Farum

Sociológico

\section{Forum Sociológico}

Série II

28 | 2016

Interculturalidade e educação

\title{
O poder de uma história : encontros e desencontros culturais
}

The power of a story: cultural matches and mismatches

\section{Ana Catarina Mendes Garcia}

\section{OpenEdition}

1 Journals

Edição electrónica

URL: https://journals.openedition.org/sociologico/1406

DOI: $10.4000 /$ sociologico.1406

ISSN: 2182-7427

Editora

CICS.NOVA - Centro Interdisciplinar de Ciências Sociais da Universidade Nova de Lisboa

Refêrencia eletrónica

Ana Catarina Mendes Garcia, «O poder de uma história : encontros e desencontros culturais», Forum Sociológico [Online], 28 | 2016, posto online no dia 31 dezembro 2016, consultado o 29 março 2022.

URL: http://journals.openedition.org/sociologico/1406 ; DOI: https://doi.org/10.4000/sociologico.1406 


\title{
LES FACES CACHÉES DE LA DIVERSITÉ RELIGIEUSE ET LEUR UTILISATION POTENTIELLE EN ÉDUCATION INTERCULTURELLE \\ AS FACES OCULTAS DA DIVERSIDADE RELIGIOSA E SEU USO POTENCIAL NA EDUCAÇÃO INTERCULTURAL \\ THE HIDDEN FACES OF RELIGIOUS DIVERSITY AND THEIR POTENTIAL USE IN INTERCULTURAL EDUCATION
}

\author{
Abdeljalil Akkari \\ Université de Genève, Faculté de Psychologie et des Sciences de l'Education, Section des sciences de l'éducation
}

\begin{abstract}
Résumé
Cet article propose une approche novatrice pour la prise en compte de la diversité religieuse à l'école. Cette approche est basée sur une double posture. D'une part, nous proposons de mettre en évidence la diversité interne de chaque religion afin de rompre avec la conception essentialiste habituelle de la religion. D'autre part, il nous semble important d'insister sur les liens existants entre différentes religions et croyances. Afin de permettre une prise en compte de la diversité religieuse en éducation interculturelle, les acteurs de l'éducation doivent être ouvert au relativisme et syncrétisme religieux et se distancer de la tendance de chaque religion à croire détenir la vérité spirituelle absolue.
\end{abstract}

Mots clefs: religion, diversité, relativisme culturel, interculturel

\section{Resumo}

Este artigo oferece uma abordagem inovadora para o reconhecimento da diversidade religiosa nas escolas. Esta abordagem baseia-se numa postura dupla. Em primeiro lugar, propomos destacar a diversidade interna de cada religião para romper com a concepção essencialista usual de religião. Em segundo lugar, parece-nos importante sublinhar as ligações entre as diferentes religiões e crenças. Para permitir a consideração da religião na educação intercultural, os actores educacionais devem estar abertos ao relativismo e ao sincretismo, distanciando-se da tendência de cada religião em acreditar ser portadora da verdade espiritual absoluta.

Palavras-chave: religião, diversidade, relativismo cultural, interculturalidade

\section{Abstract}

This article offers an innovative approach to the recognition of religious diversity in schools. This approach is based on a double position. First, we propose to highlight the internal diversity of each religion to break away the usual essentialist conception of religion. Secondly, it seems important to emphasize the links between different religions and beliefs. To allow inclusion of religion in intercultural education, religious education stakeholders must be open to relativism and syncretism and distance themselves from the tendency of each religion to believe in possession of absolute spiritual truth.

Keywords: religion, diversity, cultural relativism, interculturalism 


\section{Introduction}

Malgré un processus de sécularisation existant et plus ou moins prononcé selon les contextes, la religion constitue l'une des dimensions importantes dans la construction de l'identité culturelle des élèves. Toutefois, la prise en compte de la diversité religieuse à l'école apparaît comme une option difficile dans les approches interculturelles. En effet, l'une des conditions préalables de l'interculturalité est une certaine ouverture au relativisme culturel. Or, les croyances religieuses sont rarement relatives. Chaque religion postule une certaine supériorité par rapport aux autres. De surcroit, les institutions religieuses se considèrent habituellement comme les garantes de l'orthodoxie et de la pureté de la religion.

Ce texte a pour principal objectif d'explorer I'utilisation potentielle de la diversité religieuse en éducation. Ce que nous entendons par diversité religieuse est à la fois la diversité interne d'une religion mais aussi le rapport plus ou moins distant qu'un individu entretient avec la tradition religieuse héritée de sa famille ou de sa culture d'origine. Dans la première partie de l'article, nous mettons en évidence une certaine confusion terminologique à propos de la diversité religieuse. Dans la deuxième partie, nous nous focalisons sur la diversité interne de chaque religion. La troisième partie du texte propose une analyse de la porosité des frontières entre religions. La quatrième partie met en évidence la difficile séparation entre apprentissage d'une religion et apprentissage sur la religion. La cinquième partie présente l'expérience suisse en matière de prise en compte de la diversité religieuse et de production de manuels scolaires visant à développer l'apprentissage sur les religions.

\section{Confusion terminologique}

Dans le discours actuel aussi bien scientifique que courant, diversité religieuse et pluralisme religieux ont tendance à être interchangeables. Durant d'autres périodes, ces deux termes avaient des sens différents. La diversité religieuse est utilisée pour souligner le fait qu'il y a des différences significatives entre les religions au niveau doctrinal, social ou politique mais aussi des différences en matière de pratique religieuse. Le pluralisme religieux souligne l'acceptation, la reconnaissance ou même I'encouragement de la diversité et la vision que la "salvation/libération" peut être trouvée dans toutes les religions ou en dehors (Meister, 2011).

Le terme pluralisme religieux, d'un emploi désormais courant, renvoie donc à des réalités et des significations diverses. Le pluralisme religieux peut être utilisé dans des sens descriptif et évaluatif. Certains observateurs estiment qu'il faut parler de diversité religieuse pour qualifier la réalité empiri- que tout en gardant le pluralisme qui représente une manière bien spécifique de prendre en compte cette diversité. Le pluralisme serait donc la réponse idéologique et politique à la diversité empirique fondée sur le respect mutuel des différents systèmes religieux et/ou culturels et sur la visée d'une coexistence pacifique des diverses religions.

Au-delà de cette distinction, le problème de la définition du pluralisme religieux, de son extension et de ses limites, reste entier. Il est important d'observer tout d'abord que le pluralisme religieux s'oppose au "monisme religieux" où une seule religion détient la légitimité religieuse et plus largement symbolique. C'est un principe politique qui appartient à la pensée politique occidentale contemporaine. On peut d'ailleurs noter que si la référence au pluralisme religieux est positivement connotée dans ces sociétés, le terme ne fait pas véritablement sens dans nombre de pays si ce n'est, assez souvent, comme renvoyant à une imposition idéologique et politique du "Nord" sur le "Sud" (Champion, 1999).

Cadge, Levitt et Smilde (2011) considèrent que le "Christocentrisme (Christocentrism)" renvoie à I'omniprésence du christianisme comme une référence hégémonique dans le débat sur la diversité religieuse. Ces auteurs estiment que cette référence est récente dans I'histoire humaine dans la mesure où elle fait référence au christianisme tel qu'il est pratiqué en Europe et aux États-Unis. Smilde et May (2010) cités par Cadge et al. (2011) observent qu'entre 1978 et 2007, plus de $50 \%$ des articles publiés dans les revues de sociologie de la religion se réfèrent au christianisme. Poulson et Campbell (2010) cités par Cadge et al. (2011) ont trouvé que $82 \%$ des articles publiés entre 2001 et 2008 dans la revue Journal for the Scientific Study of Religion and Sociology of Religion traitent du christianisme. A mesure que les chercheurs traitent dans leurs travaux du phénomène religieux dans d'autres régions du monde, les limites de ce christocentrisme apparaissent clairement.

Par ailleurs, I'utilisation du terme foi, qui est plus courante en anglais qu'en français, est également problématique. L'utilisation de foi et religion comme termes interchangeables interpelle. Selon (Nesbitt, 2009), le recours aux termes "tradition religieuse" ou "foi traditionnelle" marque une tentative pour rendre I'approche à la religion moins essentialiste.

Wolfs, El Boudamoussi, De Coster et Baillet (2007) ont montré la difficulté de traduction du terme "Laïcité" dans différentes langues. En anglais, bien que le terme "Laicity" existe, c'est la notion de "secularism" qui est utilisée. En arabe, la laïcité est traduite par plusieurs termes comme madania (civil, citadin, urbain) ou almania, dont la racine est "monde". Les connotations attachées au concept almania, sont globalement négatives (confusion avec I'athéisme, l'anti-religion) dans cette langue. 
Toute cette confusion terminologique a amené Debray (2002: 171-172) à proposer le terme consensuel de "fait religieux":

\footnotetext{
"Ne le nions pas: le fait religieux est de bonne diplomatie. L'expression a de l'emploi parce qu'elle est commode, et d'une neutralité peu compromettante. Elle ne privilégie aucune confession en particulier. Chacun en a sa part et tous l'ont tout entier. Le laïque soupçonneux d'une possible contrebande spiritualiste excusera le religieux par le fait, qui force, dit-on, à s'incliner. Et le croyant réticent devant toute réduction positiviste d'une foi vivante excusera le fait parce que religieux: qu'importe le flacon, pourvu qu'on ait le mystère ! L'alliage des deux mots neutralise I'un par l'autre. Le positif par le mystique, et vice-versa. Aussi bien confessants et libres-penseurs y trouvent-ils leur compte, sans y regarder de près. Chacun peut faire sa part du feu (...)."
}

Dans la première partie de ce texte, nous avons tenté de synthétiser les principales discussions autour des termes utilisés pour aborder la diversité religieuse. Non seulement le passage d'une langue à une autre est redoutable pour de nombreux termes mais la connotation historique et politique des mots est différentes selon les contextes nationaux. Cette confusion terminologique n'est pas favorable à une opérationnalisation dans les écoles, les classes et les programmes scolaires de l'éducation à la diversité religieuse. Elle exige de la part des chercheurs et éducateurs un effort particulier pour définir des termes communs et analyser leur perception par les acteurs scolaires.

\section{Les faces cachées de la diversité interne de chaque religion}

La prise en compte de la religion dans l'espace scolaire constitue un véritable défi si l'on se place dans une véritable posture interculturelle. En effet, la religion, comme un ${ }^{1}$ des éléments caractéristiques d'un groupe, d'un peuple ou d'un individu est souvent abordée dans une perspective monolithique, essentialiste et rigide. Autrement dit, I'identité religieuse est habituellement définie au singulier. Dans ce texte, nous adopterons une posture différente en postulant l'utilité pédagogique d'une prise en compte à l'école de la diversité interne constitutive de toute religion. Comme le dit Willaime (2007), la diversité culturelle est fortement présente au sein des univers religieux eux-mêmes et que la gestion de cette diversité est un défi, y compris pour les acteurs religieux.

En premier lieu, il nous semble important de noter que la baisse de la pratique religieuse produit une diversité accrue du rapport des individus et des groupes à la religion et au sacré. Des peuples et des territoires qui pouvaient se réclamer d'une tradition religieuse dominante ne peuvent plus se prévaloir d'un tel fait aujourd'hui. Ainsi, l'Europe occidentale pouvait se prévaloir d'une tradition chrétienne dominante ne peut plus le faire aujourd'hui sans risque d'exclure une partie de sa population. La sécularisation d'une bonne partie de la population européenne nous incite à considérer une pluralité des rapports des citoyens européens de souche au christianisme. La même constatation peut être effectuée à des degrés variables par rapport à d'autres religions dans le monde.

Ainsi, un sondage récent montre que seulement $4 \%$ des parents français retiennent la foi religieuse parmi les qualités importantes à encourager chez les enfants (Hervieu-Léger, 2002). Cela ne signifie pas que les parents en question soient étrangers à toute croyance religieuse. Mais la croyance religieuse est devenue à leurs yeux une affaire de choix personnel, et elle n'est pas nécessairement associée à l'obligation de la transmettre et de socialiser les enfants dans la religion. Le thème du "droit au choix" laissé aux enfants leur permet aux parents de justifier leur refus, explicite ou implicite, d'agir eux-mêmes comme agents de la transmission religieuse. Cette posture parentale répond au souhait exprimé par les jeunes de "pouvoir choisir leur religion" (s'ils jugent nécessaire d'en avoir une) en fonction de l'affinité qu'ils ressentent avec telle ou telle tradition religieuse. Toutes les institutions de socialisation sont confrontées à cette profonde mutation culturelle qui place la notion de choix au centre de l'identité individuelle. Toutefois, I'ébranlement qui en résulte est d'autant plus sérieux, dans le cas des institutions religieuses, que la transmission engage - au-delà de la reproduction institutionnelle proprement dite - ce qui est au principe même de leur existence, à savoir la continuité de la mémoire qui les fonde. Toute religion implique en effet une mobilisation permanente de la mémoire collective (Hervieu-Léger, 2002).

En second lieu, il importe de souligner que la plupart des religions intègrent des interprétations diverses d'une même foi. Si on prend à titre d'exemple l'islam, en plus des courants majoritaires et connus que constituent le sunnisme et le shiisme, on trouve d'autres doctrines qui se réclament de I'islam. Pour signaler les plus importantes, on peut mentionner les ibâdites, les druzes, les sikhs², les ahmadis et les bahaïs et bien d'autres courants plus ou moins proches du soufisme. Mourad (2016) rappelle à juste titre ce qui est aujourd'hui trop souvent oublié. L'islam n'est pas un, mais multiple et pluriel. Pourtant, les musulmans comme les non-musulmans finissent par croire que le seul vrai, c'est I'islam littéraliste et conservateur, celui 
des intégristes qui sont aujourd'hui au cœur de I'actualité internationale.

De surcroit, on peut souligner que la conception différente selon les contextes du rapport Etat et Religion vont avoir des conséquences sur la manière dont on assume l'identité religieuse individuelle et collective. Ainsi, trois pays majoritairement musulmans, la Turquie, I'Iran et I'Indonésie vont produire des versions bien différentes de l'identité religieuse. En Turquie, I'Etat est officiellement laïc et garantit la liberté du culte même si le pays est actuellement dirigé par un parti islamo-conservateur. En Iran, le shiisme est religion d'Etat et la liberté religieuse est restreinte. Ce qui a par exemple contraint la communauté bahaïe à l'exil. L'Indonésie, pays musulman le plus peuplé au monde, a préservé la diversité des cultures religieuses (Pohl, 2006).

En troisième lieu, il faut tenir compte de la position des familles par rapport à la religion. Cela est d'autant plus important lorsque I'on parle de diversité religieuse à l'école. Les familles ne se situent pas dans une dualité entre ceux qui ont une foi inébranlable et ceux qui n'ont aucune croyance religieuse. Les familles se situent sur un continuum entre ces deux positions extrêmes. Ce que les historiens et anthropologues ont observé, c'est qu'il y a différentes façons de vivre sa foi ou sa conviction religieuse dans toutes les religions: de la simple bigoterie au mysticisme, de la pratique assidue à la vague conviction, de la ferveur illuminée au simple confort moral (Dortier, 2004).

En quatrième lieu, les migrations internationales font voyager les religions et les croyances au-delà de leur zone d'influence d'origine. L'acculturation des migrants et leur rapport à la religion d'origine varient énormément entre d'une part une sorte de zèle de fidélité aux croyances religieuses d'origine, et d'autre part une posture plus souple qui rejoint la sécularisation de certaines sociétés d'accueil ${ }^{3}$. Là aussi les catégorisations duales ne font pas de sens. L'origine religieuse des migrants en Europe, particulièrement pour les musulmans, présentée souvent comme un frein à l'intégration ou à l'insertion dans les sociétés d'accueil élude la diversité du rapport des migrants au religieux.

Une étude récente réalisée en Suisse montre par exemple le détachement relatif des jeunes de toutes les religions par rapport à la pratique régulière de la prière même si des pratiquants assidus existent (Jödicke, 2011).

En cinquième lieu, les cas des mariages interreligieux sont de plus en plus fréquents. Il n'est pas rare de trouver des enfants vivant dans des familles se réclamant de plusieurs religions ou d'aucune. Comment classer d'une manière absolu ces enfants dans des cases religieuses hermétiques alors que le rapport aux religions dans ce type de familles est complexe et diversifié.
Si la religion est historiquement indissociable de la culture, il ne faut pas oublier la pluralité des identités des individus dans le monde contemporain. Cette pluralité culturelle croissante, que ce soit en raison de l'influence des médias et des contacts interculturels, de la mobilité sociale, des mariages mixtes ou des quêtes spirituelles, incite les éducateurs à considérer également leur propre pluralité (Nesbitt, 2009). Jackson (2012: 6) appelle à ce propos à présenter les traditions religieuses dans leurs diversités: "Religious traditions should be presented, not as homogeneous and bounded systems, but in ways that recognise diversity within religions and the uniqueness of each member, as well as the fact that each person is subject to various influences".

La diversité cachée à l'intérieur de chaque religion nous oblige à une plus grande souplesse quand on introduit des contenus scolaires à propos des religions. L'essentialisme religieux consistant à définir une religion par rapport à des références historiques ou théologiques stables ou rigides n'est pas compatible avec une approche interculturelle de l'éducation. Albera (2005) propose par exemple à propos de I'islam de "déplacer le regard", compte tenu notamment de l'immense littérature actuelle qui tend à le stigmatiser et à l'essentialiser comme une religion et une culture menaçante voire "terroriste". Il propose de partir au contraire de l'islam local (d'en bas) afin de démentir les postulats d'homogénéité, de cohérence et de fixité qui caractérisent la posture culturaliste.

Enfin, la distinction entre "spiritualité" et "religion" nous semble constituer une piste de réflexion intéressante pour l'éducation à la diversité religieuse. La distinction entre "spiritualité" et "religion" n'est pas aisée. La littérature est d'ailleurs partagée à ce propos. Fry et al. (2005) estime que la spiritualité est nécessaire pour la religion, mais la religion n'est pas nécessaire pour la spiritualité. Selon Zinnbauer, Pargament, Cole, Rye, Butter, Belavichet et al. (1997), la spiritualité renvoie à l'expérience individuelle avec la transcendance alors que la "religion" se réfère à la théologie instituée et aux rites religieux. Ces mêmes auteurs ont mis en évidence le fait que les individus très religieux différencient peu ces deux termes alors que les personnes qui s'identifient comme spirituelles voyaient moins de recouvrement.

\section{Traversées de frontières et partage entre les religions}

Non seulement chaque religion est diverse comme nous avons tenté de le démontrer dans la section précédente de ce texte. Mais de surcroit, les religions partagent de nombreux éléments communs. Les points communs peuvent être abordés à deux niveaux. Tout d'abord au niveau théologique ou doctrinaire. Ensuite au niveau des pratiques religieuses 
locales des croyants. Nous n'aborderons pas le premier niveau qui a fait l'objet de nombreuses études notamment en ce qui concernent le socle commun des religions monothéistes (Poupard, 1984). A ce propos, la recherche archéologique démontre une certaine continuité entre les conceptions religieuses de l'Egypte pharaonique et les religions monothéistes apparues toutes les trois au Moyen-Orient. Nous nous concentrons sur la traversée des frontières religieuses dans les pratiques locales.

Les ouvrages récents consacrés à la fréquentation des mêmes sanctuaires par des fidèles appartenant à des groupes confessionnels différents mettent en évidence un phénomène largement méconnu de traversées des frontières entre religions (Albera, 2011; Albera e Couroucli, 2009; Ben-Ami, 1990). Habituellement, les actes de dévotion sont perçus comme des moyens de souligner et de renforcer les frontières religieuses, alors que certains d'entre eux prennent place dans des conditions de rapprochement pacifique et de mixité religieuse autour de lieux saints "partagés". Ils font apparaître des relations originales, qui étaient restées jusqu'ici très largement invisibles, surtout à une époque où sont plus volontiers mis en avant les affrontements religieux. La mixité religieuse, cette aberration pour tout intégrisme religieux, s'avère pourtant être un phénomène traditionnel, surtout dans les régions orientales de la Méditerranée, là où les populations ont su s'adapter à une instabilité de la vie politique et religieuse depuis de nombreux siècles. Les processus d'homogénéisation induits, au cours du $X X^{\text {ème }}$ siècle, par des nationalismes agressifs ont substantiellement modifié le terreau propice à ces échanges religieux (Albera e Couroucli, 2009).

Ces franchissements des frontières religieuses se reproduisent au fil des siècles. Aussi bien au Moyen Âge qu'à l'époque moderne, des sources variées attestent une présence importante de pèlerinages à deux, voire à plusieurs faces. Une contribution importante à la compréhension de ces phénomènes d'imbrication interreligieuse (qui se manifestent souvent sous la forme de pèlerinages mixtes) vient des recherches sur les relations entre chrétiens et musulmans dans I'Empire ottoman. Les relations entre ces deux groupes religieux à I'intérieur de I'Empire étaient souvent symbiotiques. Aussi bien les chrétiens que les musulmans étaient prêts à adresser leurs demandes à un sanctuaire administré par une autre religion, si ce dernier possédait une réputation d'efficacité (Albera, 2011).

Pour prendre en compte les dépassements des frontières religieuses, qui semblent souvent pratiqués par les pèlerins, il faut déplacer l'épicentre de I'analyse: des systèmes religieux institués vers les conceptions et les pratiques religieuses des acteurs locaux. Aussi bien les frontières entre religions et confessions que les identités religieuses ne sont pas des données établies et univoques, par rapport auxquelles on peut décrire les fréquentations plurielles comme des écarts, des anomalies, des extravagances (au sens littéral du terme). Il s'agit plutôt d'interroger les processus stratifiés qui génèrent des espaces religieux, dont les différentes situations d'équilibre dépendent des négociations et des confrontations entre des groupes et des acteurs variés (Albera, 2011).

Il nous semble important de souligner qu'il y a une forte valeur ajoutée éducative possible à ce constat fait par la recherche sur la traversée des frontières entre les religions. Paradoxalement, les dernières décennies ont été propices à la montée de l'intolérance religieuse justement dans les endroits connus par la présence de pèlerinage mixtes. Quand on sait que les bosniaques musulmans et les croates catholiques fréquentent actuellement des classes séparées en Bosnie, on se rend bien compte de I'intérêt potentiels à présenter aux élèves des deux communautés les pèlerinages mixtes comme un héritage historique commun.

\section{Une nécessaire mais difficile séparation entre "apprendre une religion et apprendre sur une religion"}

Durant les dernières années, de nombreux spécialistes de l'éducation ont postulé la nécessaire séparation entre éducation religieuse et connaissance des religions et du fait religieux (American Academy of Religion et al., 2007; Chidester, 2003; Jackson, 1997; Jackson, 2008). Cependant, cette séparation qui est nécessaire dans une perspective interculturelle est plus difficile à opérationnaliser qu'il n'y paraît. Tout d'abord, il y a un problème de personnes et acteurs impliquées dans l'éducation religieuse et de crédibilité de cette séparation. Il est en effet naïf de croire qu'un enseignant d'éducation religieuse ou de catéchisme peut se convertir par une baguette magique en anthropologue des religions. Par ailleurs, le fait que certaines religions soient considérées comme "religion d'Etat" fait que la présence de la religion à l'école n'est pas anodine ou fortuite et équivaut à une présence hégémonique par rapport aux autres croyances. C'est ce que nous avons constaté dans un projet sur la diversité religieuse en Méditerranée occidentale développé dans le cadre du Conseil de l'Europe, et pas seulement dans les pays de tradition islamique (Maroc et Algérie) mais aussi dans certains pays européens comme l'Espagne et I'Italie.

Comme l'a montré Sunier (2009) dans le cas de la Grande-Bretagne, la plupart du temps, l'éducation religieuse est chrétienne. La réforme éducative de 1988 (Education Reform Act) stipule que les programmes scolaires doivent "refléter les traditions religieuses qui sont principalement chrétiennes". Bien que la société britannique soit devenue multiconfes- 
sionnelle en raison de l'immigration, les prétentions hégémoniques chrétiennes sont toujours faites en prescrivant la "nature essentiellement chrétienne" pour l'éducation religieuse qui est considérée comme un guide moral dans une société multiculturelle.

L'étude de Phipps et Matkin (2009) a exploré le degré d'ouverture à la diversité religieuse parmi des étudiants pratiquants. Cette étude a mis en évidence les tensions internes vécus par les sujets qui peuvent être schématisées par trois axes: (a) ouverture vers d'autres vérités religieuses vs maintien d'une religion qui détient la vérité unique, (b) ouverture vers d'autres religions vs crainte du rejet et (c) ouverture vers autrui vs maintien de la foi.

On voit donc bien la difficulté pour une personne d'enseigner la diversité religieuse à l'école en oubliant sa foi et ses propres convictions religieuses. La problématique dépasse largement le débat sur le curriculum et l'école. La question de savoir si la religion est un élément soustractif ou additif en éducation multiculturelle est important. Dans le cas de la religion en éducation multiculturelle, il y a une préoccupation inavouée que l'exposition aux autres religions peut ouvrir la porte au questionnement de sa propre foi d'origine, ou même remplacer sa vérité religieuse par une autre ou même encourager le prosélytisme (Wilson, 2006).

Leirvik (2011) distingue à juste titre trois niveaux du dialogue interreligieux: (a) entre les élèves dans la classe, (b) entre les croyants dans la société civile, et (c) entre l'État et les communautés/ minorités religieuses. Chacun de ces dialogues possède ses propres caractéristiques et sa dynamique propre. Les conversations officielles entre I'Etat et les représentants des communautés religieuses est le plus vulnérable des dialogues interreligieux.

L'enseignement à propos des religions à l'école illustre aussi combien il est difficile de tracer une ligne de démarcation entre les dialogues initiés par I'Etat et ceux portés par la société civile. D'une part, I'éducation religieuse dans une école publique tiendra compte des idéologies nationales et des priorités de I'Etat, soit en donnant un espace à l'enseignement confessionnel ou en ouvrant une arène pour un apprentissage interreligieux. D'autre part, l'école ne peut contribuer à la formation de bons citoyens que si les élèves sont également autorisés à communiquer librement et de façon critique à propos de la religion et de l'éthique. On peut donc légitimement se demander si cela est possible dans le cadre d'un enseignement confessionnel de la religion à l'école. Comme l'a bien montré Simmons (2006), enseigner à propos des religions peut devenir très vide un guidage spirituel.

Rey (2009: 5) rappelle que vivre et apprendre ensemble la diversité religieuse à l'école n'est pas facile et exige une posture de modération et d'équilibre:
"Les élèves pourront exprimer à l'école leurs appartenances religieuses, leurs convictions non religieuses ou leur indifférence par rapport à ces questions, tout en respectant les sentiments de leurs camarades, c'est-à-dire avec modération, sans ostentation ni prosélytisme. On évitera pourtant, même avec I'intention louable de valoriser la diversité, de leur imposer de l'extérieur une identité figée (une étiquette donnée par des enseignants ou des camarades...) ou de souligner des différences et de provoquer des stigmatisations. Les circonstances sont nombreuses, dans lesquelles les jeunes peuvent se sentir mal à I'aise. Mettre le doigt sur leurs sentiments, leur situation ou leurs différences (de convictions religieuses notamment, mais aussi d'habitat, de langue familiale, de handicap...) alors qu'ils/elles souhaitent être 'comme les autres' peut représenter un manque de tact dommageable. Dans les situations délicates, des techniques de distanciation permettront aux élèves de réfléchir et d'entrer en dialogue dans des domaines qui les touchent sans être impliqués directement ni embarrassés. Un esprit d'ouverture leur donnera la possibilité d'exprimer leurs sentiments si et quand ils le souhaitent".

L'approche interprétative proposée par Jackson (2012) peut constituer une bonne piste de travail. Elle vise à augmenter la connaissance et la compréhension des religions sans promouvoir ni miner les croyances religieuses. C'est un défi qui nécessite un périlleux équilibre.

L'Académie Américaine de Religion fait la distinction entre "enseigner à propos des religions" et "endoctrinement religieux" à l'école. La première approche exige que:

- L'approche de l'école à la religion est académique et pas confessionnelle.

- L'école peut s'efforcer de sensibiliser les élèves aux religions, mais ne devrait pas insister pour l'acceptation par les élèves d'aucune religion.

- L'école peut parrainer l'étude de la religion, mais ne peut pas parrainer la pratique de la religion.

- L'école peut exposer les élèves à une diversité de points de vue religieux, mais ne peut pas imposer aucun point de vue particulier.

- L'école peut renseigner sur toutes les religions, mais ne peut pas promouvoir ou dénigrer une religion.

- L'école peut informer l'élève sur les différentes croyances, mais ne devrait pas chercher à le conformer à aucune croyance particulière. 
La laïcité de l'école et à l'école peut à cet égard représenter une option intéressante pour opérationnaliser au niveau pédagogique la nécessité

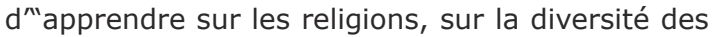
religions et sur la diversité de chaque religion". Elle a I'avantage de rendre possible une dissociation entre le cognitif et le spirituel. La laïcité peut également être une option intéressante pour les sociétés ou le religieux risque d'avoir une posture hégémonique sur le politique.

L'islam est à ce propos un cas intéressant et complexe. Ainsi, Carré (1993) s'est inscrit en faux contre la plupart des historiens qui prétendaient l'impossible séparation entre pouvoir politique et religion en Islam. Il a mis en évidence le fait que tous les pouvoirs dans le monde musulman ont utilisé des logiques parfaitement temporelles et se sont contentés de négocier leur légitimité religieuse avec un corps d'oulémas (religieux) plus ou moins domestiqués. Carré (1993) s'inscrit en faux contre la thèse courante consistant à opposer, un christianisme par origine essentiellement apolitique portant en germe l'évolution laïque et individualiste des sociétés occidentales contemporaines, et islam associant à jamais religion et Etat, spirituel et temporel, bref, essentiellement théocratique. Carré (1993) met en évidence une même tendance à la séparation des pouvoirs dans la pensée politique des deux religions monothéistes.

La singularité française par rapport à la diversité religieuse est aussi intéressante à analyser. Pour la France, républicaine, tout ce qui relève des croyances est de l'ordre du privé; elles sont donc respectées mais leur enseignement ne peut trouver de place à l'école publique (Borne, 2004).

En Europe, même quand une nation se reconnaît volontiers dans le message porté par une Eglise, cette Eglise ne porte plus le monopole du religieux à l'école. L'exemple grec est édifiant: le but de l'éducation, dit l'article 16 de la constitution du pays, est de développer "la conscience nationale et religieuse des Grecs". On sait que I'orthodoxie définit la nation elle-même, on observe cependant les débuts d'une sécularisation de cet enseignement, la possibilité de s'y soustraire, l'autorisation donnée aux non orthodoxes d'assurer l'enseignement religieux (Borne, 2004).

Si la plupart des spécialistes de l'éducation estiment qu'il n'est plus possible de laisser la religion à l'écart de l'école, ils divergent de la manière de la prendre en charge la connaissance des faits religieux et la manière de le présenter aux élèves.

\section{Expérience suisse en matière de prise en compte de la diversité religieuse à l'école}

Dans cette partie du texte, l'objectif est de voir comment la diversité religieuse est traitée à l'école suisse et dans les manuels scolaires d'éducation religieuse utilisés actuellement dans le pays. Tout d'abord, il faut rappeler qu'en Suisse chaque canton est souverain en matière de politique éducative, et donc nous avons 22 systèmes éducatifs et donc 22 manières de considérer la présence de la religion à l'école. D'une manière générale, on peut souligner que la posture de l'école par rapport à la religion se caractérise par la souplesse comme en témoigne l'extrait ci-dessous d'une publication officielle du Canton de Fribourg:

\footnotetext{
"L'école fribourgeoise, fondée sur le respect des droits fondamentaux de la personne, admet le port du foulard islamique par les élèves. Elle considère, en effet, que la prise en compte de cette prescription religieuse pour les élèves, pour autant qu'elle soit exempte de tout prosélytisme, ne met pas en cause un enseignement ordonné et efficace. Par contre, le port d'un voile dissimulant le visage n'est pas autorisé, car il empêche une bonne communication (non verbale) entre les élèves et I'enseignante; La singularisation d'une élève par un signe aussi visible que le port du foulard peut toutefois gêner son intégration et sa socialisation dans le groupe classe et plus largement, au sein de l'ensemble des élèves fréquentant un établissement scolaire. Comme pour les autres questions, les enseignants-e-s et les directions d'école sont invités à attirer l'attention des parents concernés sur cette problématique et à ouvrir le dialogue." (Direction de l'Instruction Publique, 2010: 15)
}

En Suisse, I'enseignement public sur le thème de la religion introduit dans plusieurs cantons se veut explicitement non religieux. Sa désignation dans le curriculum scolaire est diverse. "Ethique et religions" en Argovie, "Ethique et cultures religieuses" à Fribourg, "Histoire de la religion" au Tessin, "Histoire biblique-cultures religieuses" dans le canton de Vaud, "Religion et culture" à Zurich, et "Enseignement des cultures religieuses et humanistes" à Neuchâtel. Ce cours a le plus souvent un caractère obligatoire. La plupart des cantons qui I'expérimentent le considèrent comme une branche à part entière à l'exception du canton de Neuchâtel où cet enseignement est intégré dans la branche histoire. Certains groupes se sont fortement opposés à cet enseignement. Pour les associations de libres penseurs, cet enseignement menace la liberté de religion. Les groupements à tendance évangélique, eux, le considèrent comme une ingérence inadmissible dans l'éducation dispensée par les parents. Par ailleurs, certains représentants de minorités, craignent que des contenus religieux chrétiens puissent être transmis sous couvert d'enseignement public (Jödicke, 2011). 
L'expérience de la maison d'édition Enbiro dans la production d'ouvrages scolaires est intéressante. C'est une maison d'édition scolaire à but non lucratif fondée en 1967 par plusieurs cantons romands. Trois principes constituent la base de la ligne éditoriale d'Enbiro: (a) refus de toute forme de prosélytisme; (b) approche religieusement neutre, avant tout historique et culturelle et (c) respect des convictions de chacun. L'analyse des manuels utilisés dans I'enseignement sur les religions en Suisse montre qu'il y a une approche plurielle des religions. Ainsi, une brochure destinée aux élèves de la $5^{\text {ème }}$ année du primaire intitulée "Aux origines du monde" est divisée en trois parties: (1) Le regard scientifique sur I'histoire de l'univers, (2) La création du monde selon la bible, et (3) Des récits d'origine des quatre coins du monde.

Toutefois, on peut observer, qu'il y a peu de place accordée la diversité interne de chaque religion et à la traversée des frontières religieuses. Comment peut-on interpréter cette situation? Il nous semble que la plupart des rédacteurs des manuels se donnent comme priorité de palier à l'ignorance du fait religieux à l'école et à la nécessité de refléter la diversité religieuse des élèves. Par conséquent, ils utilisent une approche, certes ouverte mais aussi encyclopédique se contentant souvent par présenter les rites institutionnalisés dans une religion. Par exemple dans le cas de l'islam, on présente les 5 piliers (achahada, les prières quotidiennes, le Ramadan, l'aumône (zakat) et le pèlerinage à la Mecque). Cela transmet à l'élève un message d'étanchéité entre les religions et de monolithisme à l'intérieur d'une même religion.

La suisse représente un cas intéressant dans la prise en compte de la diversité religieuse à l'école. En effet, à la présence du protestantisme et du catholicisme comme religions dominantes dans de nombreux cantons du pays, s'ajoute l'arrivée de l'islam et d'autres religions dans le sillage des migrations internationales. Les cantons peuvent reconnaître une religion comme élément central de leur identité (par exemple les cantons du Valais et Fribourg) ou bien revendiquer une posture laïque comme les cantons de Neuchâtel ou Genève. Toutefois, même dans ce dernier, le fait religieux à l'école est abordé:

\footnotetext{
"Affirmer que l'école est laïque, c'est rappeler cette neutralité de l'État à l'égard de toutes les Églises et de toutes les confessions, mais ce n'est pas nier et esquiver la thématisation et la compréhension du fait religieux dans sa diversité. Affirmer que l'école est laïque, ce n'est pas renoncer à aborder certains sujets susceptibles de fâcher, car ce serait oublier les missions de l'école - qui sont notamment d'éveiller chez l'élève le respect d'autrui et la
}

tolérance dont notre société a grandement besoin. Une laïcité de l'ignorance, une laïcité qui a peur du contact et qui fuit le débat, fait le jeu de I'obscurantisme. Ce n'est pas cette laïcité que nous voulons." (DIP, 2016: 3)

\section{Conclusion}

Dans cet article, nous avons tenté de présenter une approche originale de la prise en compte de la diversité religieuse à l'école. Une première option possible est de préserver l'école de tout débat sur la religion en adoptant la laïcité comme principe de base. Cependant, il y a deux limites à cette posture. En effet, la laïcité n'empêche pas la religion de rentrer à l'école par le biais d'accommodements historiques avec les Etats nations et les religions. L'exemple de la France est ce propos édifiant. Le fait que l'Etat prône la laïcité comme valeur suprême de la république ne l'empêche pas de financer largement les écoles privées catholiques. L'autre limite concerne I'irruption du fait religieux par l'intermédiaire des élèves, en particulier ceux issus de l'immigration. Une école laïque peut être confrontée au port des signes religieux à l'école ou à des tensions entre pratique religieuse et apprentissages scolaires.

Nous avons proposé une option consistant à insister à l'école sur la diversité interne de chaque religion et sur la traversée salutaire des frontières entre différentes religions. L'avantage de cette option est d'initier à travers les approches interculturelles les élèves à un relativisme religieux. L'altérité religieuse devient possible et contribue à lutter contre un enfermement dans des certitudes religieuses transmise par la famille ou les communautés d'origine. Evidemment, cette option rencontrera des résistances, en particulier de la part de certaines familles soucieuses d'une socialisation mono-religieuse et des organisations religieuses.

En bref, il nous paraît important de ne pas laisser le fait religieux uniquement en dehors de l'école ou entre les mains des représentants officiels des différentes religions. Il est utile de l'intégrer dans les approches interculturelles par l'intermédiaire d'une posture qui privilégie les doutes, les emprunts, la traversée des frontières par rapport à la tentation de voir les religions et ceux qui les pratiquent comme un tout, cohérent et immuable.

Depuis plusieurs décennies, I'éducation multiculturelle/interculturelle a émergé dans de nombreux systèmes éducatifs dans le monde. Néanmoins, une confusion conceptuelle demeure et limite l'impact pédagogique de cette innovation majeure. Cette confusion est encore plus grande quand il s'agit de traiter la question de la diversité religieuse. Nous avons proposé dans ce texte de traiter les religions d'une manière peu habituelle. D'une part, en insistant sur la diversité interne 
d'une même religion et des liens et emprunts qu'elle maintient avec d'autres. D'autre part, nous avons mis en évidence les rapports flexibles et mouvants que les individus maintiennent avec la religion. Dans un monde où les migrations et les interactions sont globales, il nous paraît primordiale de sortir de la vision essentialiste et figée du rapport des individus et des groupes aux religions.

\section{Notes}

1 Dans les approches interculturelles, il est important de considérer la religion comme l'un des éléments des identités culturelles individuelles et collectives et non pas comme l'élément principal. La même précaution s'applique à la langue, l'appartenance sociale, l'ethnicité, la race.

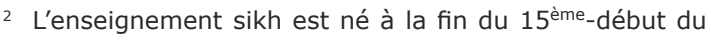
$16^{\text {ème }}$ siècle dans le contexte des rencontres entre l'islam et sa mystique soufie, et une partie de la spiritualité hindoue.

3 A ce niveau, le contraste entre certains pays européens et les Etats-Unis est saisissant. Dans les pays européens, la pratique religieuse des migrants, surtout les musulmans, est considérée habituellement comme un frein à I'intégration. Aux Etats-Unis, pays fondé au départ sur les valeurs religieuses des colons anglo-saxons protestants de la côte-est, la religion est traditionnellement considérée comme une ressource pour l'intégration des migrants. Les attentats du 11 septembre 2001 ont quelque peu changé cette donne en ce qui concerne les États-Unis.

\section{Références}

ALBERA, D. (2005), "Déplacer le regard", in P. Sant Cassia et T. Fabre (ed.), Entre Europe et Méditerranée. Les défis et les peurs, [Fait suite à la rencontre de Durham en juillet 2004], Arles, Actes Sud, pp. 125-155.

ALBERA, D. (2011), "La mixité religieuse dans les pèlerinages", Archives de Sciences Sociales des Religions, 3 (155), pp. 109-129.

ALBERA, D. and M. Couroucli (orgs.) (2009), Religions traversées. Lieux saints partagés entre chrétiens, musulmans et juifs en Méditerranée, Arles, Actes Sud.

AMERICAN ACADEMY OF RELIGION, et al. (2007), Religion in the Public School Curriculum, Syracuse, NY, Syracuse University.

BEN-AMI, I. (1990), Cultes des saints et pèlerinages judéo-musulmans au Maroc, Paris, Miasonneuve et Larose.

BORNE, D. (2004), "Ecole et religion", Revue Internationale d'Éducation Sèvres, 36, pp. 21-25.

CADGE, W.; P. Levitt and D. Smilde (2011), "DeCentering and Re-Centering: Rethinking Concepts and Methods in the Sociological Study of Religion", Journal for the Scientific Study of Religion, 50 (3), pp. 437-449.

CARRÉ, O. (1993), L'islam laïque ou le retour à la Grande tradition, Paris, Arman Colin.
CHAMPION, F. (1999), "De la diversité des pluralismes religieux", International Journal on Multicultural Societies, 1 (2), pp. 40-54.

CHIDESTER, D. (2003), "Religion Education in South Africa: Teaching and Learning about Religion, Religions, and Religious Diversity", British Journal of Religious Education, 25 (4), pp. 261-278.

DEBRAY, R. (2002), "Qu'est-ce qu'un fait religieux?", Etudes, 3973, pp. 169-180.

DIP (2016), La Laïcité à l'école, Genève, Département de I'Instruction Publique de la Culture et du Sport.

DIRECTION DE L'INSTRUCTION PUBLIQUE (2010), Diversité religieuse et culturelle à l'école. Recommandations à l'usage du corps enseignant et des autorités scolaires, Fribourg.

DORTIER, J.-F. (org.) (2004), Le dictionnaire des sciences humaines, Paris, Editions Sciences Humaines.

FRY, L. W. ; S. Vitucci and M. Cedillo (2005), "Spiritual Leadership and Army Transformation: Theory, Measurement, and Establishing a Baseline", The Leadership Quarterly, 16, pp. 835-862.

HERVIEU-LEGER, D. (2002), "La transmission des identités religieuses", Sciences Humaines, 36, pp. 56-59.

JACKSON, R. (1997), Religious Education: An Interpretive Approach, London, Hodder and Stoughton.

JACKSON, R. (2008), "Teaching about Religions in the Public Sphere: European Policy Initiatives and the Interpretive Approach", Numen, 55 (2-3), pp. 151-182.

JACKSON, R. (2012), Studying Religions: The Interpretative Approach in Brief, Oslo, European Wergeland Centre.

JÖDICKE, A. (2011), La religion à l'école, la religiosité des jeunes et les processus de différenciation dans une Suisse plurielle, Berne, FNRS.

LEIRVIK, O. (2011), "Philosophies of Interreligious Dialogue: Practice in Search of Theory", Approaching Religion, 1 (1), pp. 16-24.

MEISTER, C. (2011), The Oxford Handbook of Religious Diversity, New York, Oxford University Press.

MOURAD, S. (2016), La Mosaïque de I'islam. Entretien sur le Coran avec Perry Anderson, Paris, Institut d'Études Avancées de Nantes-Fayard.

NESBITT, E. (2009), "Ethnography, Religion and Intercultural Education", in M. de Souza (ed.), International Handbook of the Religious, Moral and Spiritual Dimensions in Education, Springer, pp. 387-398.

PHIPPS, K. A. and G. S. Matkin (2009), Exploring Openness to Religious. Diversity Among Leadership Students Who are Religious, Midwest Academy of Management.

POHL, F. (2006), "Islamic Education and Civil Society: Reflections on the Pesantren Tradition in Contemporary Indonesia", Comparative Education Review, 50 (3), pp. 389-409. 
POULSON, Stephen C. and Colin Campbel (2010), "Isomorphism, Institutional Parochialism, and the Sociology of Religion", American Sociologist, 41 (1), pp. 31-47.

POUPARD, P. (org.) (1984), Le Dictionnaire des religions, Paris, PUF.

REY, M. (2009), Des politiques éducatives à leur mise en œuvre dans les institutions scolaires (et autres contextes éducatifs), Strasbourg, Conseil de l'Europe.

SMILDE, David and Matthew May (2010), "The EmerGing Strong Program in the Sociology of Religion", in Social Science Research Council Working Paper, GA, University of Georgia, Department of Sociology.

SIMMONS, J. K. (2006), "Vanishing Boundaries: When Teaching About Religion Becomes Spiritual Guidance in the Classroom", Teaching Theology \& Religion, 9 (1), pp. 37-43.

SUNIER, T. (2009), "Teaching the Nation: Religious and Ethnic Diversity at State Schools in Britain and the Netherlands", Teachers College Record, 111 (6), pp. 1555-1581.
WILLAIME, J.-P. (2007), "Réflexions et propositions suite à la consultation des communautés religieuses par le Conseil de l'Europe", Colloque Lisbonne (22-24 juin 2007) Promouvoir le dialogue interculturel: enjeux et perspectives du Conseil de I'Europe, Conseil de l'Europe / Centre Nord-Sud. http://www.coe.int/t/dg4/intercultural/source/ Willaime_Lisboa2_FR.doc

WILSON, H. S. (2006), "The Other/Neighbor in World Religions: An Exploration from a Multicultural Education Perspective", in F. Salili e H. Rumjajn (eds.), Religion in Multicultural Education, Greenwich, CT, Information Age Publishing, pp. 9-40.

WOLFS, J.-L.; S. El Boudamoussi; L. De Coster and D. Baillet (2007), "Comment le concept de 'laïcité' est-il compris et interprété en dehors de la francophonie? Analyse comparative des traductions du terme laïcité en anglais, arabe, espagnol et néerlandais", Education Comparée, 61, pp. 97-113.

ZINNBAUER, B. J.; K. I. Pargament; B. Cole; M. S. Rye; E. M. Butter; T.G. Belavich, et al. (1997), "Religion and Spirituality: Unfuzzying the Fuzzy", Journal for the Scientific Study of Religion, (36), pp. 549-64.

Recebido a 27/05/2016. Aceite para publicação a 07/09/2016.

Abdeljalil Akkari (abdeljalil.akkari@unige.ch). Université de Genève, Faculté de Psychologie et des Sciences de I'Education, Section des sciences de l'éducation. 42, boulevard du Pont-d'Arve, CH -1211 Genève 4, Switzerland. 